\title{
Caracterização do Sistema Éter Diglicidílico do Bisfenol A / Poliaminas Alifáticas
}

\author{
Filiberto González-Garcia, Eduardo Miguez, Bluma G. Soares \\ Instituto de Macromoléculas Prof. Eloisa Mano, UFRJ
}

\begin{abstract}
Resumo: O sistema epoxídico constituído pelo éter diglicidílico do bisfenol A (DGEBA), produto DER 331 com endurecedor 24 comercializados pela Dow Química do Brasil foi caracterizado por métodos espectroscópicos, calorimétricos e de determinação de grupos funcionais. O endurecedor 24 é constituído por trietilenotetramina (60\% molar) e uma mistura de etilenoaminas polifuncionais com estruturas lineares, ramificadas e cíclicas. O equivalente epoxídico da resina resultou de $187 \mathrm{~g} \mathrm{eq}^{-1}$. A funcionalidade média, o equivalente de amina, e a concentração do endurecedor que satisfaz a estequiometria, corresponde a 5,54,27,5 $\mathrm{g} \mathrm{eq}^{-1}$, e $14,7 \mathrm{phr}$, respectivamente. O sistema epoxídico manifestou uma entalpia de reação de $486 \mathrm{~J} \mathrm{~g}^{-1}\left(106,6 \mathrm{~kJ} \mathrm{eq}^{-1}\right)$, e uma temperatura de transição vítrea $\left(\mathrm{Tg}_{\infty}\right)$ de $124{ }^{\circ} \mathrm{C}$. O trabalho apresenta ainda um programa de cura em duas etapas onde se alcança a conversão máxima, e se apresenta o diagrama de transformações conversão vs temperatura (CTT).
\end{abstract}

Palavras-chave: Éter diglicidílico do bisfenol A (DGEBA), trietilenotetramina (TETA), diagrama de transformações conversão vs temperatura (CTT).

\section{Characterization of Diglycidyl Ether of Bisphenol A / Aliphatic Polyamines Systems}

\begin{abstract}
Diglycidyl ether of the bisphenol A (DGEBA), product DER 331 and hardener 24 system marketed by Chemical Dow of Brazil were characterized by spectroscopic, calorimetric and determination of functional groups methods. The hardener 24 is constituted by triethylenetetramine $(60 \%$ molar $)$ and a mixture of ethylene polyfunctional amines with linear, ramified and cyclic structures. The epoxy equivalent of the resin corresponds to $187 \mathrm{~g} \mathrm{eq}^{-1}$. The functionality average, equivalent amine, and the concentration of the hardener that satisfy the stoichiometry corresponds to 5.54, $27.5 \mathrm{~g} \mathrm{eq}^{-1}$, and $14.7 \mathrm{phr}$, respectively. The epoxy system displayed an enthalpy of reaction of $486 \mathrm{~J} \mathrm{~g} \mathrm{~g}^{-1}\left(106.6 \mathrm{~kJ} \mathrm{eq}^{-1}\right)$, and a glass transition temperature $\left(\mathrm{Tg}_{\mathrm{\gamma}}\right)$ of $124{ }^{\circ} \mathrm{C}$. Also presented in this work is a cure program in two stages where the maximum conversion was reached.
\end{abstract}

Keywords: Diglycidyl ether of bisphenol - A (DGEBA), triethylenetetramine (TETA), diagram conversion time temperature (CTT).

\section{Introdução}

As resinas epoxídicas são materiais termorrígidos amplamente utilizados em adesivos, matrizes para compósitos, materiais elétricos e materiais para recobrimentos, entre outras aplicações. Isto é resultado de suas excelentes propriedades adesivas, mecânicas, térmicas e elétricas, assim como da relativa facilidade no processamento dos artefatos. Quando estes materiais são destinados para aplicações especiais que solicitam alta durabilidade, é comum a revisão dos parâmetros da formulação para atingir propriedades otimizadas. Os principais parâmetros incluem a utilização de uma proporção adequada de resina e endurecedor, um programa de cura adequado que assegure a conversão máxima e um tempo de manipulação tal que garanta uma correta utilização da formulação.

Dentre as várias famílias de endurecedores, as mais co- nhecidas e estudadas são as do tipo amina. Esta família é constituída por numerosos compostos polifuncionais com diferentes estruturas químicas, que contêm em comum a presença de grupos amina do tipo, primária, secundária ou terciária. Particularmente os sistemas que utilizam endurecedores do tipo amina alifática funcionam à temperatura ambiente, sendo amplamente utilizados para formulações adesivas porque permitem um tempo de manipulação adequado que garante a correta aplicação do adesivo ${ }^{[1-3]}$. Estes sistemas são geralmente bi-componente já que os produtos comerciais se apresentam em duas embalagens, um deles contendo a resina e o outro o endurecedor, como os componentes ativos mais importantes da formulação.

O objetivo do trabalho é obter informação dos parâmetros do sistema constituído pelo éter diglicidílico do bisfenol A (DGEBA), produto DER 331, e o endurecedor 24, comercializados pela Dow Química S.A. do Brasil. A com-

Autor para correspondência: Filiberto González Garcia, Instituto Politécnico, UERJ, Rua Alberto Rangel s/n - Vila Nova, CEP: 28630-050, Nova Friburgo, RJ. E-mail: fili@iprj.uerj.br. 
posição química e equivalente amina desse endurecedor não são fornecidas pelo fabricante. Porém, acredita-se que essas informações são de alta relevância para a utilização deste sistema como adesivo para reparo de dutos de aço utilizados para o transporte de petróleo e gás natural. Este trabalho inclui a determinação da proporção dos componentes que garante a estequiometria e também apresenta um procedimento simples para determinação da conversão máxima, além do diagrama de transformações do sistema. Além disto, a caracterização completa do endurecedor 24 , incluindo a determinação da funcionalidade média, equivalente amina, e conteúdo de nitrogênio do tipo amina primário, secundário e terciário, é apresentada.

\section{Experimental}

\section{Materiais}

Resina epoxídica do tipo éter diglicidílico do bisfenol A (DGEBA), produto DER 331, foi desidratada a vácuo a $80^{\circ} \mathrm{C}$ até desaparecimento das bolhas e endurecedor 24 (Dow Química S.A. do Brasil S.A.), usado como recebido. Um produto de grau comercial à base de trietilenotetramina (TETA) (60\%, da ACROS) contendo uma mistura de poliaminas alifáticas foi utilizado para fins comparativos.

\section{Ressonância magnética nuclear (RMN)}

Os espectros de ressonância magnética nuclear de carbono-13 $\left(\mathrm{RMN}-{ }^{13} \mathrm{C}\right)$ foram obtidos em um Espectrômetro de $300 \mathrm{MHz}$ - VARIAN Mercury-300 em solução de $\mathrm{CDCl}_{3}$ à temperatura ambiente operando a 75,4 MHz. Os deslocamentos químicos foram reportados em ppm referidos ao TMS como referência interna. Os parâmetros espectrais utilizados foram: tempo de aquisição $1,5 \mathrm{~s}$; largura espectral $18 \mathrm{kHz}$; pulso $\left(90^{\circ}\right) 35 \mu \mathrm{s}$, número de transientes 4000 , intervalo entre os pulsos (dl) $1 \mathrm{~s}$.

\section{Concentração de grupos funcionais}

O equivalente epoxídico (eq.epoxídico) foi determinado por titulação química por método volumétrico usando ácido bromídrico gerado "in situ", segundo as especificações da norma ASTM D 1652. Os resultados são a média de pelo menos três determinações. Além deste método, o equivalente epoxídico foi também determinado por dois métodos espectroscópicos: um deles utiliza a espectroscopia na região do infravermelho ${ }^{[1]}$ e outro a espectroscopia de ressonância magnética nuclear de hidrogênio ${ }^{[4]}$. O método de espectroscopia na região do infravermelho é baseado no desaparecimento da banda de absorção em $910 \mathrm{~cm}^{-1}$ que é característica do grupo epoxídico, referida à banda de absorção em 1600 $\mathrm{cm}^{-1}$ que permanece constante. $\mathrm{O}$ método de RMN consiste em obter a relação da área do sinal a 7,0 ppm relativo aos hidrogênios aromáticos e a área dos sinais em 2,8 e 3,4 ppm dos hidrogênios do anel epoxídico.

O equivalente de amina (eq.amina) do endurecedor 24 , foi determinado por dois métodos diferentes de titulação química usando técnica potenciométrica: o primeiro utiliza so- lução de ácido bromídrico em ácido acético glacial, segundo as especificações da norma ISO 9702:1996(E), e o outro baseia-se na diminuição do $\mathrm{pH}$ em meio aquoso de uma solução do endurecedor pela adições de diferentes volumes de uma solução de ácido clorídrico padronizada. Os resultados dos métodos de titulação são a média de pelo menos três determinações. Além destes métodos de titulação, o equivalente de amina do endurecedor foi determinado por método calorimétrico utilizando a calorimetria diferencial de varredura (DSC). Para isto, foram realizadas experiências dinâmicas utilizando diferentes concentrações de endurecedor. Todas as experiências foram realizadas em um equipamento PerkinElmer, modelo DSC 7, sob atmosfera de nitrogênio $(20 \mathrm{ml}$ $\mathrm{min}^{-1}$ ) com taxa de aquecimento de $10{ }^{\circ} \mathrm{C} \mathrm{min}^{-1} \mathrm{em}$ um intervalo de temperatura de 30 a $200{ }^{\circ} \mathrm{C}$.

\section{Determinação da funcionalidade}

A funcionalidade média do endurecedor 24 foi determinada a partir da razão de gelificação crítica, determinada a partir da equação de Macosko e Miller ${ }^{[5]}$ (equação 1). Para isto, foram preparadas diferentes misturas de resina epoxídica e endurecedor (excesso de grupos epoxídicos), que foram submetidas a $70{ }^{\circ} \mathrm{C}$ durante 48 horas, e posteriormente testadas por ensaios de solubilidade em dioxano. A determinação consiste em encontrar a concentração mínima, utilizando excesso de grupos epoxídicos nas misturas que conduz à iminente gelificação, ou seja, se procura a concentração mínima que leva à obtenção de uma amostra líquida (solúvel em dioxano) no final da reação, em relação a uma concentração superior onde ocorreria a gelificação.

$$
\left.r_{c} \text { (eq.epoxídico/eq.amina }\right)=\frac{1}{[(f-1)(g-1)]}
$$

Onde $r_{c}$ : razão de gelificação crítica, f: funcionalidade da resina epoxídica, e g: funcionalidade do endurecedor.

\section{Determinação da temperatura de decomposição}

A temperatura de decomposição (Td) do sistema epoxídico usando proporções estequiométricas foi avaliada por análise termogravimétrica utilizando um equipamento Perkin-Elmer, modelo TGA 7 sob atmosfera de nitrogênio com taxa de aquecimento de $20{ }^{\circ} \mathrm{C} \mathrm{min}-1$ em um intervalo de 30 a $600{ }^{\circ} \mathrm{C}$. Considerou-se $\mathrm{Td}$, como a temperatura máxima limite antes do início da decomposição na curva termogravimétrica.

\section{Resultados e Discussão}

\section{Caracterização estrutural do endurecedor 24}

Na Tabela 1 encontram-se resumidos os dados dos espectros de ressonância magnética nuclear de carbono-13 (RMN${ }^{13} \mathrm{C}$ ) do endurecedor 24 , e do produto TETA comercializado pela ACROS, incluído para fins comparativos. Os assinalamentos dos sinais foram suportados pelo emprego de dados espectrais $\left(\mathrm{RMN}-{ }^{13} \mathrm{C}\right)$ divulgados para endurecedores do tipo amina alifática ${ }^{[6]}$, assim como pelos dados encontrados anteriormente pela combinação de técnicas cromatográficas 
Tabela 1. Análise dos espectros de $\mathrm{RMN}-{ }^{13} \mathrm{C}$.

\begin{tabular}{|c|c|c|c|c|c|c|c|c|}
\hline \multirow{3}{*}{$\mathrm{X}_{1}$} & \multicolumn{8}{|c|}{ A) Para a estrutura linear $\mathrm{X}_{1}-{ }^{1} \mathrm{CH}_{2}-{ }^{2} \mathrm{CH}_{2}-\mathrm{X}_{2}$} \\
\hline & \multicolumn{4}{|c|}{$\mathrm{C}^{(1)}$} & \multicolumn{4}{|c|}{$\mathrm{C}^{(2)}$} \\
\hline & exp..$^{(a)}$ & exp..$^{(b)}$ & $\exp .^{(c)}$ & exp..$^{(d)}$ & $\exp ^{(a)}$ & exp..$^{(b)}$ & $\exp .^{(c)}$ & exp. ${ }^{(d)}$ \\
\hline $\mathrm{NH}$ & 41,7 & 40,8 & 41,9 & 41,9 & 52,5 & 51,6 & 52,7 & 52,6 \\
\hline $\mathrm{NH}$ & 49,4 & 48,5 & 49,6 & 49,6 & 49,4 & 48,5 & 49,6 & 49,6 \\
\hline $\mathrm{N}<$ & 39,8 & 38,9 & 40,2 & 39,7 & 57,5 & 56,7 & 58,0 & 56,9 \\
\hline $\mathrm{NH}_{2}$ & 38,8 & 37,9 & 39,0 & 38,9 & 61,2 & 60,4 & 61,3 & 61,2 \\
\hline NH & 46,2 & 45,3 & 46,6 & 46,2 & 58,6 & 57,8 & 58,9 & 58,7 \\
\hline & \multicolumn{4}{|c|}{ B) Para a estrutura cíclica } & & & & \\
\hline & \multicolumn{4}{|c|}{$\mathrm{C}^{(1)}$} & \multicolumn{4}{|c|}{$\mathrm{C}^{(2)}$} \\
\hline & $\exp .^{(a)}$ & $\exp .^{(b)}$ & $\exp .^{(c)}$ & exp..$^{(d)}$ & $\exp .^{(a)}$ & exp..$^{(b)}$ & $\exp .^{(c)}$ & exp. ${ }^{(d)}$ \\
\hline$-\mathrm{N}<$ & 46,2 & 45,4 & 46,2 & 46,2 & 54,7 & 53,9 & 53,8 & 54,8 \\
\hline$-\mathrm{N}<$ & 53,3 & 52,5 & 53,8 & 53,4 & 53,3 & 52,5 & 53,8 & 53,4 \\
\hline
\end{tabular}

a) Valores experimentais para o endurecedor 24

b) Valores experimentais para o produto grau comercial TETA (ACROS)

c) Valores divulgados na referência 6

d) Valores divulgados na referência 7

e espectroscópicas (CG-EM e RMN- $\left.{ }^{13} \mathrm{C}\right)^{[7]}$ durante a caracterização de um endurecedor comercial, denominado $720 \mathrm{Y}$, que resultou estar constituído por trietilenotetramina ( $62 \%$, molar) e uma mistura de poliaminas alifáticas como as mostradas na Figura 1.

O espectro do endurecedor 24, e do produto TETA, foram muito semelhantes, e os deslocamentos químicos dos sinais observados coincidem com os dados divulgados para uma

$\begin{array}{ll}\mathrm{H}_{2} \mathrm{~N}-\left(\mathrm{CH}_{2}\right)_{2}-\mathrm{NH}-\left(\mathrm{CH}_{2}\right)_{2}-\mathrm{NH}_{2} & \text { Dietilenotriamina (DETA) } \\ \mathrm{H}_{2} \mathrm{~N}-\left(\mathrm{CH}_{2}\right)_{2}-\mathrm{NH}-\left(\mathrm{CH}_{2}\right)_{2}-\mathrm{NH}-\left(\mathrm{CH}_{2} \mathrm{k}_{2}-\mathrm{NH}\right. & \text { Trietilenotetramina (TETA) } \\ & \text { Tris-(2-aminoetil)amina (TAEA) } \\ \end{array}$

Figura 1. Componentes do endurecedor 24 (Dow Química S.A. do Brasil) e do produto de grau comercial trietilenotetramina (ACROS). família de endurecedores conhecida por polietilenopoliaminas (PEPAs), neste caso, de baixa massa molecular. As PEPAs estão constituídas por misturas de etilenoaminas polifuncionais com estruturas lineares, ramificadas e cíclicas $^{[6,7]}$.

Os sinais dos espectros foram atribuídos a carbonos metilênicos correspondentes a fragmentos estruturais de poliaminas alifáticas como os mostrados na Tabela 1. A coincidência dos dados espectrais destes dois produtos, e os encontrados anteriormente no endurecedor $720 \mathrm{Y}^{[7]}$ sugerem que a composição do endurecedor 24 é constituída por TETA, e uma mistura de etilenoaminas polifuncionais (PEPAs) como aquelas encontradas anteriormente no produto $720 \mathrm{Y}$ (ver Figura 1). A concentração aproximada de trietilenotetramina no endurecedor 24 , e no produto comercializado pela ACROS foi de 60 e $58 \%$ (molar), respectivamente. Este resultado foi obtido a partir da razão entre as áreas relativas dos sinais em 52,5, 49,4 e 41,7 ppm, correspondentes a carbonos metilênicos dos fragmentos estruturais do tipo $-\mathrm{HN}-\mathrm{CH}_{2}-$ $\mathrm{CH}_{2}-\mathrm{NH}_{2},-\mathrm{HN}-\mathrm{CH}_{2}-\mathrm{CH}_{2}-\mathrm{NH}$ - e - $\mathrm{HN}-\mathrm{CH}_{2}-\mathrm{CH}_{2}-\mathrm{NH}_{2}$, respectivamente da TETA em relação a todas as áreas dos sinais 
dos espectros. Este resultado pode estar afetado pela presença de dietilenotriamina (DETA). No entanto, a concentração de trietilenotetramina no produto comercializado pela ACROS, está em perfeito acordo com o fornecido pelo fabricante $(60 \%)$.

\section{Proporção adequada de resina e endurecedor}

As propriedades dos sistemas epoxídicos podem ser controladas por mudanças tanto de formulação como de condições de cura. Entretanto, as melhores propriedades são alcançadas quando se utilizam proporções estequiométricas. Nesta condição, o sistema consegue a estrutura de rede mais completa porque alcança a conversão máxima, e como conseqüência disto é possível obter a temperatura de transição vítrea máxima $\left(\mathrm{Tg}_{\infty}\right)$ onde o sistema vitrifica. Freqüentemente, na utilização do sistema constituído pelo éter diglicidílico do bisfenol A (DGEBA) com trietilenotetramina (TETA) utiliza-se proporção não estequiométrica (70 \%). No entanto, quando se usa proporção estequiométrica, não são observadas mudanças na resistência à tensão por um período de um ano, e se observa um ligeiro aumento nas propriedades mecânicas em flexão em condições de intemperismo ${ }^{[1]}$. Baseado nestes comportamentos gerais foi considerado que a proporção adequada de resina e endurecedor deste sistema correspondem à estequiométrica.

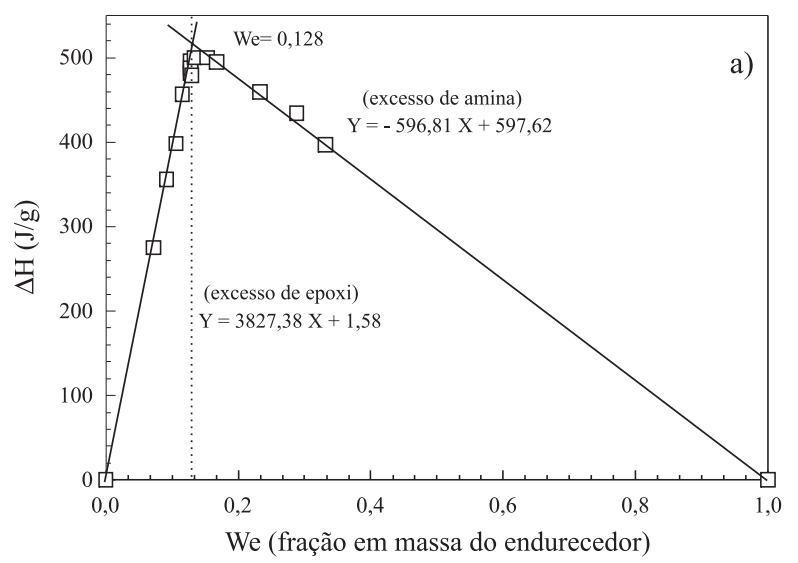

c)

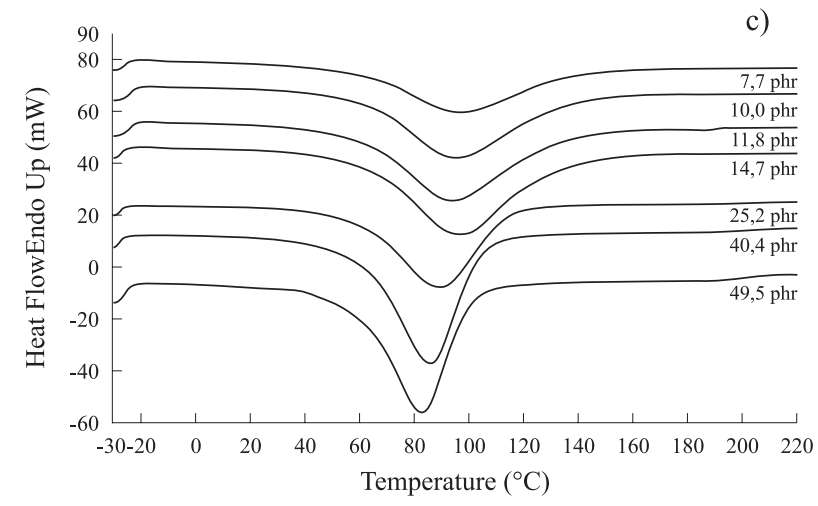

A estequiometria de um sistema epoxi-amina (um hidrogênio do tipo amina por cada grupo epoxídico) pode ser estabelecida pela determinação da concentração de grupos funcionais presentes na resina e na poliamina utilizada. Isto, muitas vezes, é determinado por métodos específicos de titulação. Nos métodos selecionados neste trabalho, as concentrações de grupos funcionais correspondem ao equivalente epoxídico, e a concentração percentual de nitrogênio (\% em massa) do tipo amina (total, primário, secundário e terciário) no endurecedor. Nas metodologias de titulação, a concentração de grupos funcionais do endurecedor necessita ser recalculada, já que estes métodos estão baseados em determinações indiretas, porque não utilizam a reação com o grupo epoxídico. Entretanto, quando se conhece o equivalente epoxídico, é possível obter de modo direto, o equivalente de amina (gramas de endurecedor que contem um equivalente de hidrogênio amínico), pelo emprego da calorimetria diferencial de varredura (DSC).

No método calorimétrico são realizadas experiências dinâmicas utilizando o sistema epoxídico com variação na concentração de endurecedor. A metodologia consiste em determinar as mudanças na entalpia de reação $(\Delta \mathrm{H})$ e na temperatura de transição vítrea $(\mathrm{Tg})$ como conseqüência da formação de diferentes estruturas de rede. Entretanto, quando se utiliza a proporção estequiométrica, os valores experimen-
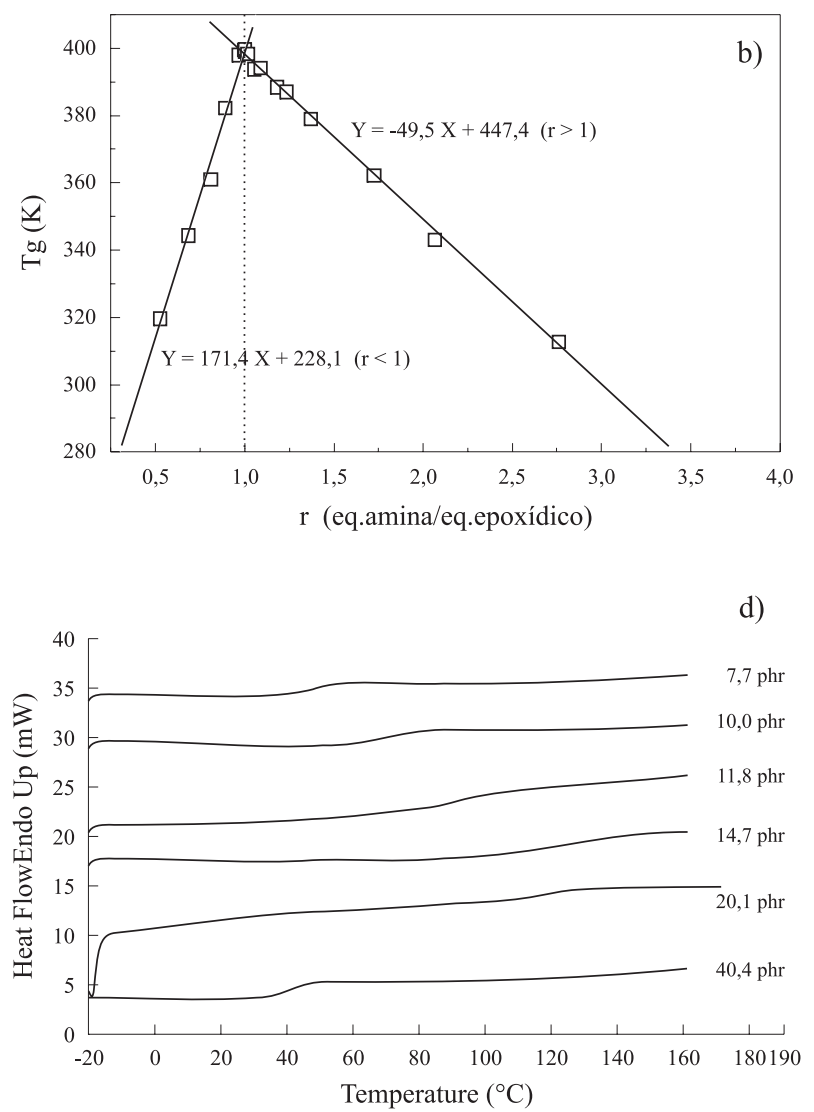

Figura 2. Determinação da proporção adequada de endurecedor 24 no sistema de resina epoxídica DER 331 por método calorimétrico (DSC). a) $\Delta H$ em função da concentração de endurecedor (We, fração em massa), b) Tg em função da concentração de endurecedor (r, razão entre os equivalentes de amina, e os equivalentes de grupos epoxídicos), c) e d) curvas calorimétricas com diferente concentração de endurecedor (phr, partes de endurecedor por cada 100 partes de resina) 
tais destas magnitudes correspondem aos valores que caracterizam o sistema epoxídico ${ }^{[8-13]}$.O resultado das experiências dinâmicas é apresentado na Figura 2. Nestas experiências, considerou-se o valor de Tg no ponto médio da transição na segunda varredura dinâmica, e o equivalente epoxídico da resina de $187 \mathrm{~g} \mathrm{eq}^{-1}$. A fração em massa do endurecedor (We) foi calculada pela equação 2 .

$$
\mathrm{We}=\frac{\mathrm{me}}{\mathrm{me}+\mathrm{mr}}
$$

Onde: me e mr correspondem à massa de endurecedor e de resina utilizada na mistura, respectivamente.

A Figura 2a mostra o comportamento experimental da entalpia de reação $(\Delta \mathrm{H})$ com diferentes concentrações do endurecedor 24. O intercepto, depois do ajuste linear dos dados experimentais corresponde a $\mathrm{We}=0,128 \pm 0,01$, que representa uma concentração de endurecedor de $14,7 \mathrm{phr}$ (gramas de endurecedor por cada 100 gramas de resina), e equivale a uma massa equivalente de $27,5 \mathrm{~g} \mathrm{eq}^{-1}$. Esta concentração satisfaz a estequiometria do sistema. Considerando que a trietilenotetramina é o componente majoritário do endurecedor 24, pode-se esperar uma fração teórica em massa de 0,115 (13,01 phr), o que não foi encontrado experimentalmente, e indica que as impurezas reativas afetam a funcionalidade. Por outro lado, o comportamento da Tg (Figura $2 b)$ utilizando diferente razão $(0,5<\mathrm{r}<4,0)$ entre os equivalentes de amina e de grupos epoxídicos na mistura, conduz à intercessão dos dados experimentais quando $r=1$. Este comportamento confirma, tanto a mudança da funcionalidade, como o valor do equivalente de amina encontrado para o endurecedor. O resultado sistemático de diferentes experiências dinâmicas para o sistema epoxídico estudado de resina DER 331 com endurecedor 24 utilizando proporção estequiométrica $(\mathrm{r}=1)$ indica que a entalpia de reação, e a temperatura de transição vítrea a conversão total $\left(\operatorname{Tg}_{\infty}\right)$ correspondem a $486 \pm 10 \mathrm{~J} \mathrm{~g}^{-1}\left(106,6 \mathrm{~kJ}^{\text {eq. }}{ }^{-1}\right)$ e $124{ }^{\circ} \mathrm{C}$, respectivamente. Estes valores estão em perfeito acordo com o divulgado para os sistemas epoxi-amina, e para os sistemas que utilizam aminas alifáticas como endurecedores, respectivamente ${ }^{[1,2,8-13]}$.

A Tabela 2 apresenta as concentrações dos grupos funcionais da resina epoxídica DER 331 e do endurecedor 24, obtidas por diferentes metodologias. O equivalente epoxídico obtido pelos três métodos corresponde a $187 \mathrm{~g} \mathrm{eq}^{-1}$. Este valor está em perfeito acordo com o divulgado pelo fabricante (182 a $192 \mathrm{~g} \mathrm{eq}^{-1}$ ). Em relação ao equivalente de amina, os métodos de titulação fornecem valores muito semelhantes quando comparados ao valor encontrado pelo método calorimétrico (DSC). Entretanto, é importante destacar que dos métodos utilizados neste trabalho para a determinação do equivalente de amina, a titulação potenciométrica em meio aquoso é a metodologia menos laboriosa, e mais accessível para qualquer laboratório de controle.

\section{Programa de cura}

Sabe-se que para os sistemas com aminas é freqüente o emprego de um programa de cura que utilize uma temperatura inicial moderada, onde pode ocorrer a gelificação e a vitrificação. Entretanto, é necessário finalizar o programa de cura a uma temperatura superior à temperatura de transição vítrea máxima $\left(\mathrm{Tg}_{\infty}\right)$ característica do sistema. O sistema constituído pela resina epoxídica do tipo DGEBA com trietilenotetramina (TETA) pode ser utilizado com êxito à temperatura ambiente na etapa inicial. Considerando que os fenômenos de gelificação e vitrificação aconteçam em um tempo de 24 horas, é possível assegurar que nestas condições, a reação não alcança a conversão máxima. Para atingir a conversão máxima, é necessário realizar uma pós-cura a uma temperatura superior à $\mathrm{Tg}_{\infty}$. Este parâmetro foi estimado a partir de análises de DSC operando em modo dinâmico em misturas de resina epoxídica e endurecedor 24 com razão estequiométrica de grupos funcionais. Nestas experiências foi encontrada uma $\operatorname{Tg}_{\infty}=124{ }^{\circ} \mathrm{C}$, indicando a necessidade da utilização de uma etapa de pós-cura a uma temperatura

Tabela 2. Concentração de grupos funcionais.

\begin{tabular}{|c|c|c|c|c|}
\hline \multicolumn{5}{|c|}{ Para a resina epoxídica DER 331 (DOW Química S.A. do Brasil) } \\
\hline \multirow{3}{*}{\multicolumn{2}{|c|}{$\begin{array}{l}\text { Equivalente epoxídico } \\
\qquad\left(\mathrm{g} \mathrm{eq}^{-1}\right)\end{array}$}} & $\begin{array}{c}\text { Norma ASTM } \\
\text { D-1652 }\end{array}$ & $\begin{array}{l}\text { Método espectroscópico } \\
(\mathrm{IV})^{[1]}\end{array}$ & $\begin{array}{c}\text { Método } \\
\text { espectroscópico (RMN- } \\
\left.{ }^{1} \mathrm{H}\right)^{[4]}\end{array}$ \\
\hline & & 187,5 & 187,3 & 187,0 \\
\hline & & \multicolumn{3}{|c|}{ Para o endurecedor 24 (DOW Química S.A. do Brasil) } \\
\hline & & $\begin{array}{c}\text { Método } \\
\text { potenciométrico } \\
\text { (Norma ISO 9702) }\end{array}$ & $\begin{array}{l}\text { Método potenciométrico } \\
\quad \text { (meio aquoso) }\end{array}$ & $\begin{array}{l}\text { Método calorimétrico } \\
\text { (DSC) }\end{array}$ \\
\hline Equiva & $\begin{array}{l}\text { e amina } \\
-1 \text { ) }\end{array}$ & 27,6 (14,7 phr) & $28,0(14,9 \mathrm{phr})$ & 27,5 (14,7 phr) \\
\hline \multicolumn{4}{|c|}{ Conteúdo de nitrogênio do tipo amina ( $\%$ em massa) ${ }^{\text {(a) }}$} & $\begin{array}{l}\text { Valor amínico total } \\
\left(\mathrm{mg} \mathrm{de} \mathrm{KOH} \mathrm{g}^{-1}\right)\end{array}$ \\
\hline primário & Secundár & terciário & Total & \multirow{2}{*}{$\begin{array}{c}1383^{(\mathrm{a})} \text { e } 1272^{(\mathrm{b})} \\
40,4^{(\mathrm{a})} \mathrm{e} 44^{(\mathrm{b})} \mathrm{g} \mathrm{eq}^{-1} \mathrm{de} \\
\mathrm{HBr}\end{array}$} \\
\hline 18,2 & 12,5 & 4,1 & 34,7 & \\
\hline
\end{tabular}




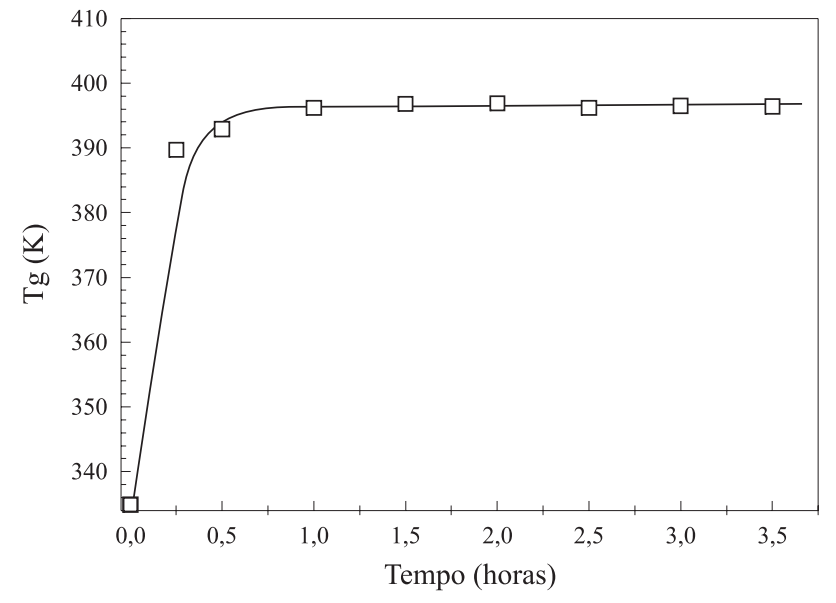

Figura 3. Comportamento da tg em função do tempo de pós-cura a $130{ }^{\circ} \mathrm{C}$ para a segunda etapa do programa de cura proposto.

superior a esta, por exemplo, $130{ }^{\circ} \mathrm{C}$. Considerando o programa de cura em duas etapas, e que a primeira etapa seja de 24 horas à temperatura ambiente, e na segunda se utilize uma temperatura de $130{ }^{\circ} \mathrm{C}$, é necessário determinar para essa segunda etapa, o tempo necessário para que a reação seja completada. Um método simples para determinar o término da reação, em condições isotérmicas, consiste em monitorar a temperatura de transição vítrea ( $\mathrm{Tg}$ ) como uma função da conversão do sistema ${ }^{[14-17]}$. Desta forma, espera-se que o aumento do tempo de reação provoque o aumento da $\mathrm{Tg}$, até alcançar o valor máximo de temperatura $\left(\mathrm{Tg}_{\infty}\right)$ onde o sistema vitrifica. O comportamento experimental da Tg do sistema parcialmente curado à temperatura ambiente (primeira etapa), e na pós-cura a $130{ }^{\circ} \mathrm{C}$ como uma função do tempo (segunda etapa), é apresentado na Figura 3.

Os resultados mostram de modo evidente que depois da primeira etapa do programa de cura o aumento do tempo de pós-cura a $130{ }^{\circ} \mathrm{C}$ provoca o aumento de $\mathrm{Tg}$, até ao valor máximo de $124{ }^{\circ} \mathrm{C}$, que corresponde à temperatura máxima onde este sistema vitrifica. Este resultado confirma que nestas condições de cura, o sistema alcança a conversão máxima.

\section{Diagrama de transformações}

Existem dois tipos de diagramas para representar as transformações que ocorrem nos materiais termorrígidos. Um corresponde ao diagrama tempo vs temperatura (TTT), proposto por J.K. Gillham e at. ${ }^{[17-18]}$, e outro, o diagrama conversão vs temperatura (CTT), divulgado por R.J.J. Williams e at. ${ }^{[19]}$. Dois tipos de transformações diferentes são características para estes materiais: a gelificação e vitrificação. Nos diagramas CTT, é necessário determinar os valores de conversão associados a estas transformações específicas. A conversão de gelificação $\left(\mathrm{x}_{\text {gel }}\right)$ depende fundamentalmente da funcionalidade dos reagentes. Em nosso sistema, o valor da funcionalidade da resina foi considerado igual a 2 , e a funcionalidade média experimental do endurecedor resultou de $5,54 \pm 0,02$, determinada a partir da razão de gelificação crítica $\left(r_{c}\right)$, correspondente a $0,220 \pm 0,001$. A partir destes dois valores do sistema é possível determinar $\mathrm{x}_{\mathrm{gel}}$ pela equação 3 ,

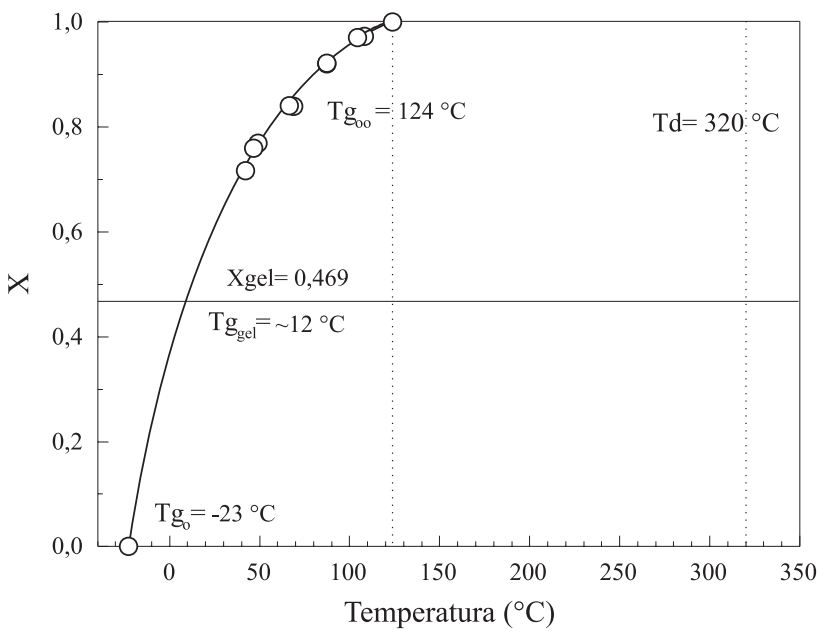

Figura 4. Diagrama de transformação CTT do sistema DER 331Endurecedor 24.

x: conversão, $\operatorname{Tg}_{\infty}$ : temperatura máxima onde o sistema vitrifica (quando $\mathrm{x}=1$ ), $\operatorname{Tg}_{\mathrm{o}}$ : temperatura do sistema no início da reação (quando $\mathrm{x}=0$ ), $\operatorname{Tg}_{\mathrm{gel}}$ : temperatura particular onde ocorrem simultaneamente a gelificação e vitrificação, xgel: conversão correspondente à gelificação, Td: temperatura de decomposição do material.

considerando que não existem efeitos de substituição (igual reatividade dos hidrogênios primário e secundário), e não ocorrem reações intramoleculares (ciclização) ${ }^{[20]}$. O valor calculado de $\mathrm{x}_{\text {gel }}$ resultou de $0,469 \pm 0,001$.

$$
\mathrm{x}_{\mathrm{gel}}=[(\mathrm{f}-1)(\mathrm{g}-1)]^{-1 / 2}
$$

Onde $\mathrm{x}_{\mathrm{gel}}$ : conversão de gelificação, f: funcionalidade da resina epoxídica, e $\mathrm{g}$ : funcionalidade do endurecedor.

A conversão ( $\mathrm{x}$ ) do sistema em condições isotérmicas, e os valores correspondentes de Tg podem ser avaliados pela combinação de experiências isotérmicas e dinâmicas (DSC). Para obter estes valores foram realizadas primeiramente experiências isotérmicas a 20,30, 50, 70 e $90{ }^{\circ} \mathrm{C}$ utilizando proporção estequiométrica. A conversão final alcançada após cada experiência isotérmica a diferente temperatura, foi determinada pela equação 4, e o valor de $\mathrm{Tg}$ correspondente foi determinado por uma experiência dinâmica posterior à isotérmica. Neste caso, considerou-se o valor de Tg no início da transição ("on set") na curva calorimétrica. As experiências dinâmicas foram realizadas, sob atmosfera de nitrogênio com taxa de aquecimento de $10{ }^{\circ} \mathrm{C} \mathrm{min}^{-1}$ em um intervalo de -60 a $200{ }^{\circ} \mathrm{C}$.

$$
\mathrm{x}=1-\frac{\Delta \mathrm{H}_{\mathrm{R}}}{\Delta \mathrm{H}_{\mathrm{T}}}
$$

Onde: $\Delta \mathrm{H}_{\mathrm{T}}$ : entalpia total do sistema de resina DER 331 com endurecedor 24 (486 J g-1), e $\Delta \mathrm{H}_{\mathrm{R}}$ : entalpia residual após a experiência isotérmica.

Os parâmetros, e as conversões (x) nos diagramas de transformações conversão vs temperatura (CTT) correspondem a: $\operatorname{Tg}_{\infty}$ : temperatura máxima onde o sistema vitrifica $(\mathrm{x}=1)$ $\mathrm{Tg}_{\mathrm{o}}$ : temperatura do sistema no início da reação $(\mathrm{x}=0)$ $\mathrm{Tg}_{\text {gel }}$ : temperatura particular onde ocorrem simultaneamente a gelificação e vitrificação $\left(\mathrm{x}_{\text {gel }}\right)$

Td: temperatura onde ocorre decomposição do material.

Tc: temperatura de cura 
Para o sistema de resina DER 331 com endurecedor 24 foram determinados os parâmetros: $\operatorname{Tg}_{\infty}=124^{\circ} \mathrm{C}, \mathrm{Tg}_{0}=-23^{\circ} \mathrm{C}$, e $\mathrm{Tg}_{\text {gel }}=\sim 12^{\circ} \mathrm{C}$ (valor aproximado obtido por extrapolação no gráfico, conversão vs temperatura, a partir de conhecer que $\mathrm{x}_{\mathrm{gel}}=$ 0,469). A temperatura de decomposição (Td) resultou $320^{\circ} \mathrm{C}$.

A Figura 4 apresenta a conversão (x) como uma função da temperatura para o sistema de estudo a que correspondem diferentes valores de Tg. A partir do diagrama CTT, é possível justificar o programa de cura proposto. Para sistemas epoxídicos está bem estabelecido que quando $\mathrm{Tg}_{\infty}>\mathrm{Tc}>$ $\operatorname{Tg}_{\text {gel }}$ a vitrificação ocorre posterior à gelificação, e quando $\mathrm{Tc}<\mathrm{Tg}_{\text {gel }}$ ocorre o contrário. Para o programa de cura proposto, em duas etapas, onde a primeira etapa corresponde à temperatura ambiente $\left(30^{\circ} \mathrm{C}\right)$ durante 24 horas, esta temperatura de cura coincide com a condição $\mathrm{Tg}_{\infty}>\mathrm{Tc}>\mathrm{Tg}_{\text {gel }}$, indicando que na primeira etapa de cura (temperatura ambiente) a gelificação ocorre primeiro à vitrificação, e que nestas condições a vitrificação ocorre a uma temperatura bem inferior à $\mathrm{Tg}_{\infty}$. Depois desta etapa inicial, para alcançar $\mathrm{Tg}_{\infty}$, é necessário utilizar uma temperatura de cura (pós-cura) igual ou superior a $124{ }^{\circ} \mathrm{C}$. Isto foi verificado neste trabalho, utilizando uma etapa de pós-cura a $130{ }^{\circ} \mathrm{C}$ durante um tempo que garanta a vitrificação do sistema (Figura 3).

\section{Conclusões}

O sistema constituído pela resina epoxídica, produto DER 331, e o endurecedor 24, comercializados pela Dow Química S.A. do Brasil foi estudado. Os resultados experimentais indicam que, o endurecedor 24 é constituído por trietilenotetramina (60\% molar) e uma mistura de etilenoaminas polifuncionais com estruturas lineares, ramificadas e cíclicas. A funcionalidade média, o equivalente de amina, e a concentração do endurecedor que satisfaz a estequiometria, corresponde a 5,54, $27,5 \mathrm{~g} \mathrm{eq}^{-1}$, e 14,7 phr, respectivamente. Este sistema manifestou uma entalpia de reação de $486 \mathrm{~J} \mathrm{~g}^{-1}\left(106,6 \mathrm{~kJ}_{\mathrm{eq}}{ }^{-1}\right)$, e uma temperatura de transição vítrea $\left(\mathrm{Tg}_{\infty}\right)$ de $124{ }^{\circ} \mathrm{C}$, valores que estão em perfeito acordo com o divulgado para os sistemas epoxi-amina e para os sistemas que utilizam aminas alifáticas como endurecedores, respectivamente. O programa de cura proposto de duas etapas: 24 horas à temperatura ambiente, e 2 horas a $130^{\circ} \mathrm{C}$, alcança a conversão máxima.

\section{Agradecimentos}

Os autores agradecem o apoio pelo Plano Nacional de Ciência e Tecnologia do Setor Petróleo e Gás Natural - CTPETRO, por meio do CNPq (CT-PETRO/CNPq) Proc. No. 500092/02-8.

\section{Referências Bibliográficas}

1. Lee, H. \& Neville, K.; "Handbook of Epoxy Resins", McGrawHill, Inc. New York, cap.7, págs.1-10; cap.4, p.19; cap.7, pag.9 e 2 (1967).

2. Pérez, R.J.; "Commercial Epoxy Resin Curing Agents”, em
Epoxy Resin Technology, editado por Paul F. Bruins, Interscience Publishers, John Wiley \& Sons, New York, p.45 (1968).

3. Scola, D.A.; "Novel Curing Agents for Epoxy Resins"; em Developments in Reinforced Plastics-4, editado por G. Pritchard, Elsevier Applied Science Publishers, New York, p.165 (1984).

4. González, F. \& Soares, B.G. - Polym. Test., 22, p.51 (2003).

5. Macosko, C.W. \& Miller, D.R. - Macromolecules, 9, p.199 (1976).

6. Bulai, A.K.; Slonim. I.Y.; Urman, Y.G.; Vakulenko, V.A. \& Chetverikova, A.T. - Polymer Science U.S.S.R., Vol.28, No.8, p.1854 (1986).

7. González, F.; Pérez, C.; Galego, N.; Martínez, R. \& Rosado, A. Revista Cubana de Química, vol.V, No.3, p.44 (1989).

8. Klute, C.H. \& Viehmann, W. - J. Appl. Polym. Sci., 5, p.86 (1961).

9. Horie, K.; Hiura, H.; Sawada, M.; Mita, I. \& Kambe, H. - J. Polym. Sci. (A-1), 8, p.1357, (1970).

10. Rozenberg, B.A.; "Kinetic, Thermodynamics and Mechanism of Reactions of Epoxy oligomers with Amines", em Epoxy Resins and Composites II, editado por K. Dušek, Springer-Verlag, Berlin Heidelberg, p.115 (1986).

11. Galy, J.; Sabra, A. \& Pascault, J.P. - Polym. Eng. Sci., 26, p.1514 (1986).

12. Vallo, C.I.; Frontini, P.M. \& Williams, R.J.J. - J. Polym.: Part B: Polym. Phys., 29, p.1503 (1991).

13. Eloundou, J.P.; Feve, M.; Harran D. Pascault, P.J. - Die. Age. Makromol. Chem., 230, p.13 (1995).

14. Babayevsky, P.G. \& Gillham, J.K. - J. Appl. Polym. Sci., 17, p.2067 (1973).

15. Gillham, J.K. \& Benci, J.A. - J. Appl. Polym. Sci., 18, p.951 (1974).

16. Gillham, J.K. - Polym. Eng. Sci., 16, p.353 (1976).

17. Wisanrakkit, G. \& Gillham, J.K.; "The Glass Transition Temperature as a Parameter for Monitoring the Isothermal Cure of an AmineCured Epoxy Resins" em Polymer Characterization Physical Property, Spectroscopic, and Chromatography Methods, editado por C.D. Craver. American Chemical Society, Washington, DC, p.143 (1990).

18. Aronhime, M.T. \& Gillham J.K. - "Time-TemperatureTransformation (TTT) Cure Diagram of Thermosetting Polymeric Systems" em Epoxy Resins and Composites II, editado por K. Dušek, Springer-Verlag, Berlin Heidelberg, p.83 (1986).

19. Adabbo, H.E. \& Williams, R.J.J. - J. Appl. Polym. Sci., 27, p.1327 (1982).

20. Miller, D.R.; Vallés E. \& Macosko, C.W. - Polym. Eng. Sci.., 19, p.272 (1979).

Enviado: 22/11/04

Reenviado: $16 / 03 / 05$

Aprovado: 24/06/05 